\title{
La calidad de las instituciones y su impacto sobre el comercio internacional
}

\author{
The quality of institutions and its impact \\ on international trade
}

\author{
Armando di Filippo *
}

\section{Resumen}

Como consecuencia de la creciente movilidad internacional de los factores productivos, las instituciones y organizaciones nacionales relevantes para la regulación del comercio internacional ya no son tan solo, y ni siquiera principalmente, las relacionadas con las aduanas que funcionan en las fronteras. Ahora involucran todo otro conjunto de instituciones y organizaciones que operan al interior de los países que participan en el flujo internacional de factores productivos. Este nuevo escenario deriva de la presencia geográfica de empresas transnacionales que al menos en parte están sometidas a la legislación interna de las naciones que las acogen. Por lo tanto la calidad de las instituciones nacionales influye directamente en el comercio internacional y, de manera más amplia, en el conjunto de las relaciones económicas internacionales.

PALABRAS ClAVE: instituciones, relaciones internacionales, negociaciones internacionales, globalización.

* Profesor del Instituto de Estudios Internacionales (armando.difilippo@gmail.com). El autor agradece la colaboración de Armando Di Filippo Pacheco, LL.M., en International Transactions and Comparative Law, Facultad de Derecho, Universidad de San Francisco, California.

Recibido el 25 de enero de 2008; aceptado el 5 de marzo de 2008. 


\section{Abstract}

As a consequence of the increasing international mobility of productive resources, national institutions and organizations relevant for the regulation of international trade are no longer, or mainly, those related with customs operating in the border. They now involve an increasing set of other institutions and organizations operating within nations that participate in this international flow of productive resources. This new scenario derives from the geographical presence of transnational corporations at least partially subject to the domestic legislation of nations where they are located. Consequently, the quality of national institutions directly affect international trade and, more generally, overall international economic relations.

KEY WORDS: institutions, international relations, international negotiations, globalization 
Armando di Filippo • La calidad de las instituciones y su impacto...

\section{INSTITUCIONES Y MERCADOS}

La noción de instituciones en su sentido amplio suele referirse a regulaciones vigentes, es decir aceptadas e internalizadas en el comportamiento de los actores a los cuales se aplican. En ocasiones no se distingue conceptualmente entre el concepto de instituciones y el de organizaciones, que son asociaciones deliberadamente creadas para cumplir ciertos fines societales. Si siguiendo a Douglass North introducimos esa distinción, tendremos tres conceptos ligados entre si: a) Instituciones entendidas como reglas formales o informales de naturaleza política, económica, o sociocultural, que son aceptadas e internalizadas por los actores sociales; b) Organizaciones (públicas, privadas, o no gubernamentales) entendidas como asociaciones deliberadamente creadas por los seres humanos para el logro de ciertos fines estratégicos en la esfera política (como los partidos políticos, las secretarías de Estado, los tribunales, o los parlamentos), económica (como las empresas) o sociocultural (como los hospitales o las escuelas); c) Actores o agentes, entendidos como individuos que actúan formando parte de organizaciones específicas o de manera independiente como ciudadanos, propietarios, o simplemente personas naturales en algún otro ámbito de la realidad (cultural, deportivo, religioso, etc.).

En el sentido señalado en a) las regulaciones gubernamentales son instituciones formales que forman parte de un Estado de derecho que puede ser preservado incluso mediante la fuerza pública monopolizada por los Estados. Una regulación gubernamental es un variado conjunto de instrumentos por el cual los gobiernos establecen requisitos que deben reunir las empresas y los ciudadanos. Las regulaciones gubernamentales, así entendidas, incluyen leyes (Constitución Política, códigos fundamentales), ordenanzas formales e informales, y reglas menores o subordinadas establecidas en toda la red de organizaciones públicas que componen el aparato gubernamental. Siguiendo en esta perspectiva formal, existe un conjunto de reglas dictadas por organizaciones intergubernamentales o no gubernamentales a los cuales los gobiernos han delegado poderes regulatorios o autoregulatorios.

Las instituciones informales incluyen reglas culturales, éticas, o incluso fórmulas de cortesía, protocolo y «buena crianza» que no están escritas o corresponden a códigos religiosos o a ciertos grupos culturalmente determinados (por ejemplo posiciones morales en materia sexual, o códigos internos de minorías étnicas). En ciertas sociedades existen comportamientos mafiosos o corruptos enquistados que no por ser informales (y obviamente ilegales) dejan de poseer vigencia e influyen en los aspectos político, económico, y sociocultural de la sociedad.

Todas estas normas internalizadas de conducta se expresan en las transacciones, entendidas como relaciones sociales de contenido económico cuyo 
costo puede ser calculado. Tal es el caso, por supuesto, de todas las transacciones de mercado. En un sentido amplio las transacciones siempre implican expectativas recíprocas de conducta asumidas por las partes que transan o contratan desde distintas posiciones institucionalizadas. Dichas posiciones son las que les otorgan su respectivo poder de contratación (propietarios de factores productivos, de bienes, de servicios, o de cualquier otro tipo de derecho, título, o valor susceptible de ser transado en el mercado). Por lo tanto, podemos reservar el vocablo transacción para las relaciones sociales de mercado en las cuales los participantes actúan como propietarios y poseen posiciones institucionales específicas en la red de derechos de propiedad. Las transacciones tienen costos y estos pueden ser examinados y calculados. En particular pueden mencionarse los costos de informarse, de negociar, de formalizar el contrato, de monitorear los compromisos y derechos adquiridos, de recurrir a los tribunales y, en caso necesario, a la fuerza pública para reclamar el cumplimiento del contrato1. También las transacciones

1 Refiriéndose a los costos de transacción, Ronald Coase, pionero en el análisis de este concepto observa: «In order to carry out a market transaction it is necessary to discover who it is that one wishes to deal with, to inform people that one wishes to deal and on what terms, to conduct negotiations leading up to a bargain, to draw up the contract, to undertake the inspection needed to make sure that the terms of the contract are being observed, and so pueden ser formales e informales, pero en estas últimas pueden eventualmente vulnerarse las redes de derechos de propiedad y, por lo tanto, introducirse ambigüedades y riesgos en las economías de mercado.

\section{INSTITUCIONES MERCADOS}

INTERNACIONALES Y REGLAS DEL JUEGO

Una manera pedagógica de entender la diferencia entre instituciones y organizaciones, sugerida especialmente por North, es encuadrarlas en, o asimilarlas a, la lógica de los juegos. Una expresión altamente formalizada, incluso en términos matemáticos, de la noción de juego ha tenido lugar a través del desarrollo de la teoría de los juegos y ha sido aplicada a la comprensión del funcionamiento de mercados altamente estilizados, por ejemplo el concepto de equilibrio de Nash, o expresiones más difundidas en los libros de texto, como el «dilema del prisionero». Veamos someramente algunas de estas ideas relativamente más formalizadas, y volvamos luego a la formulación más simplificada y pedagógica de North.

Usando una versión ultrasimplificada de la teoría de los juegos, con la misma lógica del dilema del prisionero,

on». Citando a su vez a Dahlman (1979) dice Coase: "Dahlman crystallized the concept of transaction costs by describing them as «search and information costs, bargaining and decision costs policing and enforcement costs» (Coase 1988: 6). 
es posible examinar las opciones principales en materia de política comercial aplicables a la lógica de los mercados internacionales.

La primera opción para un juego bilateral en el mercado internacional sería "ninguno coopera», es decir ambos erigen barreras que impiden el acceso del otro al propio mercado, y la recompensa por ese comportamiento es igual a cero.

La segunda opción es "ambos cooperan", y ambos obtienen una recompensa igual $a(b-c)$, en que $b$ es el beneficio derivado de acceder al mercado del otro, y c es el coste neto de abrir su propio mercado en el que $\mathrm{b}$ es un valor económico mayor que c. Si b no fuera mayor que c, se estaría negando el principio general de que el comercio internacional libre y abierto es beneficioso para todas las partes que concurren a él. Toda la teoría del comercio internacional, desde la teoría de los costos comparativos de David Ricardo en adelante, se ha dedicado a buscar pruebas científicas de que dicho principio (beneficios del comercio libre y abierto) es correcto. La existencia de organismos como la OMC implica que no basta con la libertad de comercio (entendida como "negative rule making»), sino que también se requiere construir normas aceptadas por los contratantes ("positive rule making») con base en una actitud cooperativa.

Si solo uno coopera abriendo su mercado y el otro no lo hace, entonces el que abre su mercado tiene costos de $\mathrm{c}$ y ningún beneficio $(b=0)$, o sea $(b-c=$
- c). El otro obtiene la situación opuesta es decir $(b-c=b)$; puesto que $(c=0)$.

Si se trata de un juego de un solo tiro ( «one shot game»), es decir que se juega una sola vez, entonces los jugadores pueden adoptar una posición proteccionista con lo cual la oportunidad de comercio no se abre y todo queda como estaba: $(b=0)$ y $(c=0)$. Esta será la actitud más probable en ausencia de comunicación recíproca, de negociación y entendimiento previos.

Si se trata de un juego repetido, entonces se crean incentivos para cooperar recíprocamente y adquirir compromisos, porque los acuerdos pueden ser resguardados (enforced) a través de la amenaza o la práctica efectiva de la retaliación.

Lo anterior puede resumirse en el cuadro 1 (siguiente página).

La cooperación estable es el resultado de negociaciones, genera compromisos y se convierte en una expectativa recíproca de conducta, es decir se convierte en una regla institucionalizada para las partes en el juego.

Estas reflexiones lógicas de carácter general se pueden aplicar al tipo de negociaciones que tienen lugar en la OMC y que conducen a la aprobación de reglas institucionalizadas para las partes en el juego.

Las negociaciones del tipo OMC implican un proceso compuesto de tres fases y el logro de un estado final o post-negociación (Hoekman y Kostecki, 2001: 113).

Las tres fases del proceso negociador son: la fase «catalizadora», en la cual 
Cuadro i. Juego bilateral en el mercado

INTERNACIONAL

\begin{tabular}{|c|c|c|c|c|}
\hline & & & PAÍS B & \\
\hline & & COOPERA & & NO COOPERA \\
\hline & COOPERA & $b-c, b-c$ & & $-c, b$ \\
\hline PAÍS A & & & & \\
\hline & NO COOPERA & $b,-c$ & & 0,0 \\
\hline
\end{tabular}

se definen los asuntos o materias a ser negociadas, la fase de prenegociación, en la que se discute la agenda y lo que serán las reglas básicas del proceso negociador y finalmente, la fase de negociación intergubernamental propiamente tal, que está sujeta a los parámetros establecidos en la agenda.

La post negociación o estado final corresponde a la implementación definitiva, con la incorporación de los acuerdos alcanzados en las leyes y procedimientos del país, y adquiere fuerza legal a través de las potestades administrativa, judicial y legislativa.

Este es el proceso de instalación definitiva de nuevas instituciones, entendidas como reglas vigentes, fundadas en compromisos explícitamente adquiridos, los que crean expectativas recíprocas de conducta (Hoekman y Kostecki, 2001: 100).

Pero siguiendo a North, volvemos a la visión o enfoque institucional en términos más generales, $\mathrm{y}$, sin penetrar en complejidades formales, reiteramos la distinción entre instituciones, organizaciones (personas jurídicas o asociaciones), personas naturales (seres humanos) y tecnologías. Las instituciones pueden asimilarse a reglas de un juego competitivo de equipos, las organizaciones equivalen a equipos que juegan, y las personas naturales a miembros de esos equipos. Por último, la tecnología se personifica en el know how individual y colectivo de los equipos y en el equipamiento físico que se utiliza. De esta manera, pueden introducirse conceptos como estrategia y táctica que han estado muy presentes en la teoría del management a partir de los años ochenta, paralelamente al proceso de transnacionalización empresarial que acompañó la revolución de las tecnologías de la información y el proceso de globalización.

Precisamente tenemos aquí tres conceptos analógicos que pueden ilustrarse con los rasgos definitorios de un juego de equipos, como por ejemplo el fútbol: la tecnología está personificada en la calidad de los miembros de los equipos (know how en la tarea de conducir la 
Armando di Filippo • La calidad de las instituciones y su impacto...

pelota al arco contrario) y en los instrumentos de que se valen (equipamiento deportivo); los equipos equivalen a las organizaciones y actúan concertadamente de acuerdo con reglas internas necesarias para desarrollar sus tácticas y estrategias en el campo de juego; y las reglas de juego están escritas en el reglamento de fútbol que el árbitro hace cumplir. Pero también hay reglas del juego informales, e incluso ilegales, por ejemplo cuando un defensor golpea a un delantero para evitar un gol, al precio de poder ser penalizado por el árbitro.

En el presente orden económico mundial, altamente globalizado pueden distinguirse reglas del juego que son a) acordadas por los jugadores políticos formales (Estados nacionales o supranacionales), b) jugadores económicos que cabildean y presionan a los jugadores políticos y pueden ser «mayores» (major players) como las empresas transnacionales y multinacionales o «menores» (minor players) como otras organizaciones de menor importancia económica que participan en el orden mundial), c) jugadores intergubernamentales, que son creados por los jugadores políticos y que intermedian entre estos y jugadores económicos como las organizaciones intergubernamentales que regulan y monitorean el juego de la economía global (como es el caso de la $\mathrm{OMC}$ ), y que, adicionalmente, pueden ejercer influencia crediticia y normativa sobre los jugadores políticos «menores» (en América Latina por ejemplo) como es el caso del Fondo Monetario Internacional (FMI) y el Banco Mundial.

\section{Marcos institucionales BÁSICOS}

Como lo hace notar Dani Rodrik, a mejor regulación de los mercados menor necesidad y/o posibilidad de intervención gubernamental directa, es decir menores opciones de intervencionismo estatal directo (fijación de precios mínimos o máximos, racionamiento, etc.) eventualmente arbitrario

Además, cabría agregar que a mejor regulación de los mercados menor posibilidad de que los contratantes más poderosos puedan imponer sus reglas y sus precios a los contratantes menos poderosos, haciendo uso de las fallas de mercado que los favorecen.

El referente o paradigma de mercado implícita o explícitamente presente es el modelo de equilibrio general estable bajo condiciones de competencia perfecta operando sin ningún tipo de externalidades. Como estas condiciones obviamente no existen, pueden quizá sustituirse mediante un proceso de cooperación negociado que conduzca a nuevas reglas de comportamiento que compensen las ineficiencias.

Como hacen notar Hoekman y Kostecki (2001: 110/111): «En la práctica, por supuesto, hay grupos de «lobbyistas o cabildeadores» (rent seeking groups) en cada país, gobiernos que no creen en el laissez faire, y mercados que son imperfectos. Persiguiendo objetivos nacionales, un país puede reducir el bienestar de otros países mediante la imposición de externalidades negativas sobre ellos. Una externalidad surge cuando un go- 
bierno no toma en cuenta el impacto positivo o negativo de sus acciones sobre otros países. La literatura económica sobre externalidades ha enfocado dos maneras de tratar este problema. Una requiere una autoridad central para imponer gravámenes y subsidios compensadores. La otra postula que los agentes tratarán de negociar en busca de una situación óptima en el sentido de Pareto. La primera aproximación no es muy relevante en el contexto internacional, al no existir una entidad supranacional investida del poder para adoptar esas compensaciones (en el supuesto que su monto pueda calcularse). En el corazón del segundo enfoque subyace el así llamado teorema de Coase, (en homenaje a Ronald Coase, premio Nobel de Economía): dada la existencia de derechos de propiedad vigentes (enforceable) y en la ausencia de costos de transacción, las externalidades pueden ser negociadas hasta que pueda alcanzarse una situación optimo-paretiana. En otras palabras, el mercado, -es decir la negociación- asegurará la eficiencia. En general, para que puedan negociarse reglas de comportamiento, es necesario que los jugadores tengan la expectativa de interactuar recíprocamente en un horizonte indefinido de tiempo. Esto crea incentivos para cooperar porque los acuerdos pueden ser garantizados (enforced) bajo la amenaza de retaliación».

«El teorema de Coase supone agentes perfectamente informados respecto del ámbito económico en el cual operan y también supone que pueden interactuar sin costo alguno. Esto supone información completa sobre las preferencias propias y las de los demás. En la práctica a menudo estas premisas no se verifican. Por lo tanto, usualmente no hay certeza de que un procedimiento negociador específico conducirá a un resultado eficiente. La negociación solo puede resolver un problema de externalidades si sus efectos externos son la única causa de la falla de mercados, y este no es el caso si la información es imperfecta. Sin embargo si existen instituciones que posibilitan ofertas o propuestas competitivas en materia de derechos de propiedad, una reasignación eficiente de esos derechos puede lograrse en un mundo de información incompleta».

«En asuntos internacionales, a primera vista la reasignación de derechos de propiedad o las propuestas al respecto pueden no parecer muy prácticas. Sin embargo, los derechos de propiedad existen, implícitamente definidos por las reglas de soberanía. Esto es, los Estados-nación que crean externalidades implícitamente tienen derecho a imponerlas. La existencia de esos derechos permite que puedan realizarse las negociaciones en tanto que las externalidades mutuamente negativas creadas por las políticas comerciales nacionales son el estímulo para que los países negocien. Puesto que los países interactúan continuamente, en principio los acuerdos pueden invocarse como vinculantes (enforceable) en la medida en que los incumplidores pueden identificarse y someterse a retaliación. La OMC pone gran énfasis en 
procedimientos transparentes y fiscalización o vigilancia (surveillance) recíproca, lo que facilita la identificación de los incumplidores. En determinadas condiciones, los países afectados tienen derecho a retaliar si no existe compensación por parte de los violadores del acuerdo o, si ella es inadecuada».

Nótese que por una parte el mercado puede verse como una institución porque consiste en un conjunto de reglas apoyadas en los derechos de propiedad vigentes de quienes participan en él o, también, como un mecanismo porque la competencia es su modo de operar e incluso su razón de ser.

En resumen, en última instancia lo que justifica esa institución que denominamos mercado, es que induce una asignación eficiente de los recursos a través del mecanismo de la competencia, que es su rasgo definitorio. Como la competencia dista de ser perfecta y, además existen externalidades que, al margen del mecanismo del mercado inducen ventajas o desventajas arbitrarias entre los participantes, entonces se justifica realizar negociaciones orientadas a restablecer la eficiencia del mercado, incluso mediante la modificación de los derechos de propiedad que subyacen las negociaciones. En el ámbito del mercado internacional, estas modificaciones pueden tener lugar precisamente porque las partes que negocian son Estados soberanos. Por lo tanto pueden aceptar compromisos en materia de propiedad y modificar la legislación interna en consonancia con los compromisos contraídos.
Por eso es que el último estadio de un proceso negociador en el marco de la OMC conduce a modificaciones en la legislación interna de los países firmantes, de manera de otorgar fuerza legal y certeza jurídica a los compromisos contraídos. Este procedimiento involucra y compromete a los poderes legislativo, ejecutivo y judicial de cada país.

\section{COMERCIO INTERNACIONAL Y MERCADO GLOBAL}

Ahora bien, durante un largo período los principales compromisos se relacionaban con la eliminación de las trabas al comercio de bienes en la frontera de acuerdo con los principios de no discriminación, trato nacional, y transparencia, propugnados primero por el GATT y luego por la OMC. Esto afectaba ante todo a las leyes aduaneras, en relación con medidas arancelarias o para arancelarias. Lo nuevo del escenario global actual es la creciente movilidad internacional de la tecnología, de los servicios que pueden transarse internacionalmente y del capital productivo y financiero. Por lo tanto, los compromisos que los países asumen en las negociaciones actuales de la OMC comprometen a un ámbito mucho más amplio de su legislación interna: códigos de propiedad intelectual, ambientales, laborales, de políticas de competencia y defensa del consumidor, etc.

En el mundo económico global contemporáneo, las grandes protago- 
nistas en la asignación internacional de los recursos económicos (tecnología, capital financiero, capital productivo, etc.) son las empresas transnacionales productoras de bienes (primarios y manufacturados) y servicios (técnicos $\mathrm{y}$ financieros).

Los inversionistas transnacionales aprecian en alto grado la estabilidad y transparencia de las reglas del juego en los países donde deciden hacer negocios. Ellas incluyen la existencia de normas constitucionales claras, la estabilidad y predictibilidad de instituciones políticas fundamentales tales como el Estado de derecho, de tribunales que operen de acuerdo con la ley, de poderes ejecutivos y legislativos que eviten situaciones de corrupción o arbitrariedad, y el debido respeto de los derechos de las minorías. Las dificultades experimentadas por algunas economías en transición que antes formaban parte del bloque socialista, en especial el caso de Rusia, evidencian con extrema claridad los vínculos que existen entre un marco institucional básico, establecido de acuerdo con la constitución y la estabilidad de las relaciones económicas internacionales.

Entre los derechos mas importantes que deben institucionalizarse con claridad, transparencia y justicia, se cuentan los derechos sociolaborales, y los derechos de propiedad. Ambos son esenciales para el normal desarrollo del comercio y el respeto de las disciplinas comerciales formuladas y resguardadas por la OMC. Los actores transnacionales privados, cuya importancia en las transacciones internacionales es decisiva, no persiguen sin embargo los valores de la democracia y la justicia per se. Les interesa ante todo un marco institucional que les permita evitar cambios bruscos en las reglas del juego (laborales ambientales, arancelarias, tributarias, monetarias, financieras, etc.) que más los afectan. Para asegurar la estabilidad y transparencia que reclaman los actores privados transnacionales, algunos países instalan regímenes de excepción especialmente diseñados para lograr la afluencia de inversiones y tecnología (zonas francas comerciales, zonas procesadoras de exportaciones, «paraísos» financieros y fiscales, etc.) que operan como ámbitos extraterritoriales en que rigen legislaciones especiales.

Las regulaciones macroeconómicas estables y transparentes son en general, esenciales en los planes de inversión y de comercio de los grandes actores privados (también empresas públicas) transnacionales. Las negociaciones orientadas a reducir las trabas arancelarias y no arancelarias pueden verse enormemente afectadas, contrarrestadas e incluso anuladas por convulsiones de naturaleza monetaria o financiera, como lo han demostrado las crisis ocurridas en Asia y América Latina.

\section{TÓPICOS DE DISCUSIÓN: TEORÍA E INSTITUCIONES}

El punto de partida que vincula las consideraciones anteriores con la 
Armando di Filippo • La calidad de las instituciones y su impacto...

teoría académicamente establecida del comercio internacional se ha fundado en alto grado en la creciente movilidad internacional de los factores productivos. La teoría académica establecida del comercio internacional ha supuesto en general que los factores productivos (capital, tecnología, talento empresarial, etc.) son plenamente móviles y dúctiles al interior de las fronteras nacionales (incluso aceptando condiciones de competencia perfecta como en el modelo de Heckscher y Ohlin), pero completamente inmóviles entre naciones que comercian. En eso precisamente se basa la teoría de las ventajas comparativas de las naciones que racionalizó las ventajas del comercio internacional, intersectorial, característico de los siglos 19 y primera mitad del 20 en los países de América Latina. Esta teoría intenta explicar las razones por las cuales los países latinoamericanos debían especializarse en la producción y exportación de productos primarios (actividades agrícolas de clima templado y tropical, y actividades mineroextractivas).

Después de la Segunda Guerra Mundial la teoría del comercio internacional trató de explicar por qué países similares intercambiaban internacionalmente productos similares. Se trataba de la rápida proliferación de una nueva estructura del comercio internacional, que no podía explicarse por la teoría de las ventajas comparativas (sea en su versión ricardiana o neoclásica) que se refería a países estructuralmente diferentes (por ejemplo subdesarrollados frente a desarrollados) que intercambiaban productos cualitativamente diferentes (por ejemplo, productos primarios en contraposición a manufacturas). Sin embargo, al finalizar la guerra los países desarrollados empezaron un activo comercio de bienes finales entre industrias entre empresas. Por ejemplo, los países miembros de la actual Unión Europea compraban y vendían entre sí autos de diferentes precios y modelos, fabricados al interior de cada país. De esa manera, por ejemplo, la Fiat italiana podía vender su pequeño auto utilitario (Fiat 600) no solo a varias decenas de millones de italianos sino a centenares de millones de europeos ubicados en ese nicho de demanda (franjas de precio y calidad). La explicación teórica de este tipo de comercio se encontró en el principio de los rendimientos crecientes a escala que operan en muchas funciones de producción de la industria manufacturera. El concepto de economías de escala se estudió en relación con las empresas individualmente consideradas y también en relación con sectores productivos homogéneos. Sin embargo, esta explicación teórica también se relacionaba con factores de producción (tecnología, capital productivo, talento empresarial) que al menos al comienzo se mantenían, internacionalmente inmóviles. Los automóviles Fiat se seguían fabricando en Italia y los Peugeot en Francia.

Sin embargo, lo que caracteriza decisivamente al nuevo escenario económico internacional (en especial a partir de la década de los años ochenta) es la movilidad internacional de los facto- 
res productivos. Las reglas del juego de la globalización están orientadas a facilitar y a regular el movimiento de los factores con la destacada excepción del trabajo de baja especialización. Las migraciones de trabajadores plantean otro tipo de problemas políticos, sociales y culturales que expresan la falta de adaptación de los inmigrantes (provenientes de países subdesarrollados) a los marcos institucionales de los países (desarrollados) de destino.

El segundo tópico de discusión atañe a las causas estructurales profundas que están determinando esa creciente movilidad de los factores productivos, especialmente de los que son controlados por las grandes empresas transnacionales. Las razones tecnológicas tienen que ver con el desarrollo de las tecnologías de la información y al menos parcialmente con los revolucionarios cambios en la biotecnología y la ingeniería genética que transforman las ventajas comparativas y competitivas en el campo de la agricultura. Las razones organizacionales se relacionan con las nuevas estrategias de organización y gestión de las empresas transnacionales que, muy especialmente, aprovechan y promueven esas tecnologías. Las razones institucionales tienen que ver con los cambios en las reglas del juego de la economía mundial cuya expresión más manifiesta y pertinente para nuestros fines es la nueva institucionalidad de los mercados promovida desde la OMC tras el fin de «la era GATT».

La consecuencia más decisiva de esta creciente movilidad internacional de los factores productivos es que las instituciones y organizaciones relevantes para la regulación del comercio internacional ya no son tan solo, y ni siquiera principalmente, las relacionadas con las aduanas que operan en la frontera sino que involucran a todo otro conjunto de instituciones y organizaciones que operan en el interior de los países que participan en este flujo internacional de factores productivos. Este nuevo escenario deriva de la presencia geográfica de empresas transnacionales que al menos en parte se rigen por la legislación interna de las naciones que las acogen. Por lo tanto, la calidad de las instituciones nacionales genera un impacto directo en el comercio internacional y, de manera más amplia, en el conjunto de las relaciones económicas internacionales.

\section{MOVILIDAD INTERNACIONAL DE FACTORES Y SERVICIOS}

En la actualidad, el conocimiento de la economía mundial exige tener cuidadosamente presentes las ventajas competitivas de las empresas transnacionales, ampliamente favorecidas por las tecnologías de la información que han proliferado a escala mundial desde los años 1980. La lógica o racionalidad dominante de esas ventajas competitivas será examinada en la sección siguiente de estas notas. Pero el hecho notable a considerar es que esa movilidad internacional de los factores productivos contribuye de manera 
Armando di Filippo • La calidad de las instituciones y su impacto...

significativa a transformar la dotación relativa de factores productivos, tanto a nivel específico como general.

La dotación relativa de factores productivos ya no es función de la dinámica interna de las naciones que comercian sino que se ve fuertemente impactada, en plazos relativamente cortos, por la presencia del capital productivo transnacional. Las empresas transnacionales que tienen su casa matríz en países desarrollados instalan fuertes dotaciones de capital en los países en desarrollo. En América Latina encontramos dos patrones distintivos. Por un lado, en México, Centroamérica y el Caribe proliferan empresas que aprovechan zonas francas comerciales, financieras, y manufactureras para invertir grandes capitales en esos rubros; además, se observa una creciente presencia de capital productivo la esfera de los servicios y muy particularmente en las actividades turísticas. De otro lado, en América del Sur existen inversiones en actividades primario-extractivas orientadas al mercado de los países desarrollados e inversiones manufactureras y de servicios orientadas a los mercados locales, nacionales, subregionales, o regionales de esas naciones.

Aunque en esas naciones receptoras de abundantes flujos de inversión la dotación general de capital por unidad de trabajo no cambia significativamente, eso se debe a que sigue existiendo una abrumadora mayoría de Pymes y microempresas que no participan directamente de estas nuevas inyecciones de tecnología y capital y operan mayoritariamente para el mercado interno. Esto está permitiendo que surja una nueva forma de dualismo estructural en que un número reducido de grandes empresas genera y capte los beneficios del comercio sin contribuir significativamente a la creación directa de empleos, mientras que muchas empresas pequeñas quedan al margen de esos circuitos transnacionales pero contribuyen masivamente a generar nuevos empleos.

En el mercado internacional este desplazamiento masivo de inversión productiva cambia la dotación relativa de factores productivos que se aplican directamente a la producción de bienes y servicios transables en el plano internacional.

En el escenario económico global han surgido nuevas cadenas y sistemas productivos transnacionales. Siguiendo a Michael Porter podemos reservar el nombre de cadenas productivas para los eslabones pertenecientes a una misma empresa transnacional que tiene filiales en diferentes partes del mundo, y el apelativo de sistemas productivos para las transacciones establecidas entre subsidiarias pertenecientes a diferentes transnacionales. En el primer caso tenemos comercio intrafirma y precios de transferencia. En el segundo, tenemos comercio entre partes independientes que tienen intereses diferentes pero que pertenecen a los mismos sectores, dando lugar a diferentes modalidades de comercio intrasectorial o intraindustrial. Las frecuentes fusiones y adquisiciones suelen modificar el carácter, intrafirma 
o no, del comercio intraindustrial entre las empresas.

La movilidad internacional de los factores productivos ha cambiado el significado y campo de aplicación de los principios rectores del GATT, que ya no se aplican a través de las aduanas en la frontera sino que penetran al interior de los espacios institucionales de las naciones que comercian. Por ejemplo, los principios de no discriminación (trato NMF), de trato nacional o de transparencia ya no se aplican solamente a los bienes que se comercian en la frontera sino también a las empresas que se localizan al interior de los países, o a la propiedad intelectual de ciertos productos importados que son objeto de copia o «pirateo». Estas nuevas circunstancias derivan de la revolución de las tecnologías de la información cuyo impacto en las estrategias competitivas de las empresas transnacionales analizaremos someramente en lo que sigue.

\section{TECNOLOGÍAS DE LA INFORMACIÓN}

Las tecnologías de la información han reducido dramáticamente los costos internacionales de transacción y de coordinación de las empresas transnacionales posibilitando la proliferación planetaria de los sistemas y cadenas de valor que son característicos del comercio intraindustrial e intrafirma de bienes y servicios.

En el campo de las transacciones entre partes independientes se han reducido enormemente los costos de encontrar una contraparte contractual, de negociar con ella, y de formalizar el contrato correspondiente. Para ilustrar el punto basta mencionar el comercio electrónico a través de las supercarreteras de información que proporciona la Internet. Sin embargo, es posible que hayan aumentado los costos de monitorear los compromisos contraídos y los costos judiciales y legales de hacerlos cumplir. Todos los costos mencionados dependen, entre otras cosas, de la calidad de las instituciones involucradas y en particular de las regulaciones internacionales asociadas al desempeño de la OMC.

De otro lado, los costos de coordinación de las cadenas productivas al interior del «espacio de planificación» de las empresas transnacionales se han reducido espectacularmente mediante nuevas tecnologías de organización y gestión, tales como el intercambio electrónico de información, el uso de códigos de barras, los sistemas «justo a tiempo» de manejo de los inventarios, y la aplicación de esas mismas tecnologías por las empresas de transporte, lo que abarata y acelera la posibilidad de una mejor coordinación entre subsidiarias que comparten un mismo espacio de planificación transnacional.

Estas facilidades tecnológicas posibilitan nuevas estrategias competitivas de las empresas. Este tema ha dado lugar a la proliferación de escritos económicos en el campo de la organización y la gestión, que incluyen nombres como los de Porter, Drucker, Ohmae, Yip y muchos otros. Cabe destacar aquí un 
Armando di Filippo • La calidad de las instituciones y su impacto...

planteamiento sencillo de Dunning de acuerdo con el cual se examinan algunas de esas nuevas estrategias que han surgido de manera creciente a partir de los años 1980. Según este autor las empresas persiguen ventajas estratégicas de localización, de internalización, y de monopolio tecnológico.

Las ventajas de localización apuntan a la reducción de costos medios en esferas específicas tales como la laboral, la ambiental, la energética, etc., mediante la instalación de subsidiarias en naciones o zonas francas en que estos costos son sustancialmente menores. Como es obvio, esta clase de ventajas depende en alto grado no solo de la abundancia relativa de los factores productivos involucrados sino también de las regulaciones en campos tan importantes como el laboral, el ambiental, los servicios públicos (energía por ejemplo), etc.

Las ventajas en materia de internalización apuntan a reducir los costos de transacción, a preservar los secretos tecnológicos, y a posibilitar una planificación transnacional a mayor plazo. También permiten reducir, en algunas actividades, la plantilla o nómina total de personal contratado por las empresas que (mediante fusión o absorción) se integran vertical u horizontalmente. De nuevo aquí la absorción de empresas ha estado ligada, especialmente en América Latina, a procesos de privatización de empresas públicas y al pago de la deuda mediante la cesión de estas a las partes acreedoras. Desde luego, todos estos procesos requieren que estas estrategias cuenten con el adecuado marco institucional y regulatorio en los países involucrados.

Las ventajas derivadas del monopolio tecnológico intentan aprovechar economías dinámicas de escala, absorbiendo enormes gastos fijos en investigación y desarrollo, con una masiva oferta del producto en los mercados mundiales (piénsese por ejemplo en las sucesivas versiones del Programa Windows de Microsoft). El monopolio tecnológico depende en altísimo grado de la calidad y vigencia de las instituciones y de las organizaciones. Por ejemplo, en el caso de Windows, esas ventajas exigen que se cumplan estrictas normas de sanción al pirateo en los países demandantes. También están sujetas a las normas derivadas de los regímenes y políticas de defensa de la competencia y del consumidor, para evitar que estos grandes monopolios impongan normas técnicas perjudiciales para sus competidores potenciales.

Todas estas estrategias de las empresas transnacionales tienen algún grado de regulación internacional a través de las normas de la OMC y de otras organizaciones gubernamentales. Es el caso, por ejemplo, de las normas del GATS (servicios), las TRIM (inversiones vinculadas con el comercio), las TRIP (propiedad intelectual). También operan, o podrían operar, otras regulaciones financieras, medioambientales, etc., que afectan directamente las opciones estratégicas mencionadas más arriba. 
CALIDAD DE LAS INSTITUCIONES (REGULACIONES) NACIONALES

Las normas de la OMC relativas a inversiones, servicios, propiedad intelectual, compras estatales, etc., exigen altos grados de correspondencia con las respectivas regulaciones nacionales, tales como códigos laborales y ambientales, códigos de inversiones, códigos de propiedad intelectual, legislación en materia de servicios públicos (telecomunicaciones, energía), etc. También exigen que los poderes públicos (ejecutivo, legislativo y judicial) hagan cumplir la ley y los compromisos internacionales asumidos por las naciones miembros de la OMC.

Otro tema de enorme importancia es el de las trabas burocráticas y procedimentales de organizaciones y reparticiones públicas ineficientes. En ciertos casos, pueden ser excusas para formas ocultas de proteccionismo comercial. $\mathrm{O}$, de manera más general, pueden incrementar exageradamente los costos de transacción en que se ven obligados a incurrir los inversionistas para hacer valer sus derechos patrimoniales. También aquí se requiere de normas claras, previsibles y transparentes.

Un último aspecto importante, especialmente para los negociadores nacionales ante la OMC, es el grado de comunicación y coordinación interna que pueden desarrollar con sus contrapartes de las diferentes reparticiones involucradas en los procesos que se negocian al interior de sus respectivos países. Esto incluye el asesoramiento y la información que los negociadores pueden recibir de esas reparticiones, el grado de conocimiento que tengan los funcionarios nacionales acerca de los compromisos que los negociadores, han ido asumiendo ante la OMC en nombre de sus países y, finalmente, el tipo de modificaciones organizacionales y de gestión que es preciso realizar para poder cumplir con las nuevas normativas.

\section{Conclusiones}

En ciertos casos la integración de los países al orden económico internacional se expresa poniendo marcado énfasis en la idea de desregulación, es decir, en la eliminación de las reglas de diferente orden que estaban trabando el desarrollo de las oportunidades de comercio e inversión a nivel global. Los escritos anglosajones especializados en esta orientación que pone énfasis en la desregulación pura y simple suelen llamarla «negative rule making».

Pero la calidad de las instituciones y su impacto en el comercio internacional no es fruto de un proceso de desregulación entendido como una especie de «reforma desregulatoria». Se trata de practicar una reforma regulatoria capaz de responder a los requerimientos del desarrollo de las economías latinoamericanas en el marco de los procesos de globalización en que les toca vivir. Lo que los autores anglosajones suelen llamar "positive rule making».

En resumen, el intervencionismo arbitrario de los poderes públicos en las 
Armando di Filippo • La calidad de las instituciones y su impacto...

relaciones de mercado no se combate solamente con reformas desregulatorias, sino sobre todo con instituciones de calidad suficiente como para promover el desarrollo económico al interior de los marcos regulatorios básicos que establecen las democracias capitalistas de las sociedades contemporáneas.

\section{BibLIOGRAFÍA}

Coase, Ronald (1988), The Firm, the Market, and the Law, Chicago. The University of Chicago Press.

Dahlmann, Carl (1979), The Problem of Externality, en Journal of Law and Economics 22, número 1 (abril):148.

Hoekman y Kostecki (2001), The Political Economy of the World Trading System, Oxford, Oxford University Press.

Lamy, Pascal (2005), Towards World Democracy, Londres, Policy Network, (extraído de Lamy, Pascal (2004), La democratiemonde. Pour une autre governance global, París, La république des idées).

Krugman y Obstfeld (1994), Economía Internacional, Madrid, McGraw Hill/ Interamericana de España S.A.
North, Douglas (1993), Instituciones, Cambio Institucional y Desempeño Económico, México, Fondo de Cultura Económica.

Aoki, Masahiko (2001), Toward a Comparative Institutional Analysis. Cambridge, Mass, The MIT Press.

Rodrik, Dani (2000), Trade Policy Reform as Institutional Reform, Harvard University. Documento preparado para un manual sobre Developing countries and the Next Round of WTO Negotiations, Bernard Hoekman (ed.).

Dunning, John (1993), Trade, location of economic activity and the multinational enterprise: A search for an eclectic approach, en The theory of transnational corporations. Vol. I. por John Dunning, (ed.), Londres, Nueva York, The United Nations Library on transnational corporations. Transnational Corporations and Management Division.

Porter, Michael (1991), La Ventaja Competitiva de las Naciones, Buenos Aires, Javier Vergara (ed.).

Ohmae, Kenichi (1990), La Mente del Estratega, Bogotá. McGrawHill.

Drucker, Peter (1990), Las Nuevas Realidades, Buenos Aires, Editorial Sudamericana.

Yip, George (1997), Globalización: Estrategias para obtener una ventaja competitiva internacional, Bogotá, Grupo Editorial Norma. 